\title{
Risk of avascular necrosis following short term megadose methylprednisolone treatment
}

\author{
PC Wing ${ }^{1}$, P Nance ${ }^{2}$, DG Connell ${ }^{1}$ and F Gagnon ${ }^{1}$ \\ ${ }^{1}$ Vancouver Hospital and Health Sciences Centre, Vancouver, BC, Canada; ${ }^{2}$ Health Sciences Centre Rehabilitation \\ Hospital, Winnipeg, MB, Canada
}

\begin{abstract}
We conducted a prospective cohort study to determine whether administration of large doses of the corticosteroid methylprednisolone following spinal cord injury as recommended in the National Acute Spinal Cord Injury Study-2 (NASCIS-2) protocol results in an increased incidence of avascular necrosis (AVN) of the femoral or humeral head. All subjects were patients treated by a spinal cord injury physician in an Acute Spinal Cord Injury Unit between 1989 and 1996 where some received the megadose steroids while others did not. Patients younger than 15 years and older than 75 years were excluded, as were those with any hip or shoulder disease, with pelvic fracture, or with a history of predisposition to AVN by hip dislocation, excessive alcohol consumption, previous high dose steroid use, or systemic lupus erythematosus. Screening for AVN of the femoral and humeral heads was performed at a minimum of 6 months following injury, using magnetic resonance imaging (MRI). The films were read by a radiologist blinded to the treatment protocol received by the individual subject. Among the 59 spinal cord injured patients who received steroids (age 15-64 years (mean 32 years)), five were female. Among the 32 spinal cord injured subjects who did not receive steroids (age 16 to 65 years (mean 34 years)), seven were female. There was no case of AVN found in either group. Using binomial distribution, we conclude that the true incidence of AVN among the methylprednisolone treated group is less than $5 \%(\alpha<0.05)$ and therefore continue to recommend short term $(24 \mathrm{~h})$ methylprednisolone therapy.
\end{abstract}

Keywords: methylprednisolone; osteonecrosis; adverse effects; spinal cord injury

\section{Introduction}

Steroids have long been known to improve the neurological outcome of experimental spinal cord injuries in animal studies. ${ }^{1}$ As a result, many spinal cord injury units around the world routinely administered moderate dose steroids to all patients suffering spinal cord injuries, but subsequent clinical studies failed to show any benefit. ${ }^{2,3}$ In order to demonstrate the ineffectiveness of corticosteroids, a randomized controlled study was conducted by the National Acute Spinal Cord Injury Study group (NASCIS-1). ${ }^{4}$ The treatment group received a $1000 \mathrm{mg}$ bolus of methylprednisolone (Mpred) with $1000 \mathrm{mg}$ daily for 10 days, while the control group was given a $100 \mathrm{mg}$ bolus with $100 \mathrm{mg}$ daily for 10 days. There was no significant difference in neurologic outcome between the two groups.

Most of the studies of steroids in the clinical setting did not take into consideration the pharmacodynamics and pharmacokinetics of corticosteroids which likely were responsible for the difference between human and animal study results. ${ }^{2,3}$ Clinical studies administered Mpred at $0.6-30 \mathrm{mg} \mathrm{kg}^{-1}$ within $24 \mathrm{~h}$ of injury, while

Correspondence: F Gagnon animal studies used $15-30 \mathrm{mg} \mathrm{kg}^{-1}$ within $1-4 \mathrm{~h}$ of injury.

A second randomized, controlled, double-blind study was carried out (NASCIS-2) where the control group was given a placebo, the first treatment group received a naloxone $5.4 \mathrm{mg} \mathrm{kg}^{-1}$ bolus with a $4 \mathrm{mg} \mathrm{kg}^{-1} \mathrm{hr}^{-1}$ infusion for $23 \mathrm{~h}$, and, a second treatment group received a $30 \mathrm{mg} \mathrm{kg}^{-1}$ bolus followed by a $5.4 \mathrm{mg} \mathrm{kg}^{-1} \mathrm{hr}^{-1}$ Mpred intravenous infusion for $23 \mathrm{~h}^{5,6}$ Treatment was instituted within $12 \mathrm{~h}$ of injury. Subjects who received Mpred within $8 \mathrm{~h}$ of injury showed significant improvement in neurologic outcome at 6 months post-injury compared to both the control and naloxone groups, whereas there was no difference between the control and the naloxone groups. Based on the results of this study, the majority of spinal cord injury units, including our own, now routinely follow the NASCIS-2 high-dose Mpred protocol for all spinal cord injured patients.

Following this protocol adult patients weighing 55$80 \mathrm{~kg}$ would receive between $11000 \mathrm{mg}$ and $15000 \mathrm{mg}$ of Mpred over $24 \mathrm{~h}$. Concern about these extremely high dosages caused the NASCIS-2 study group to assess the incidence of steroid-related adverse 
effects including wound infection, gastrointestinal bleeding, liver enzymes, and 'other complications'. However, there was no assessment of the potential for avascular necrosis (AVN) of the femoral or humeral head leading to hip and shoulder joint destruction, degeneration, and ultimately hip or shoulder joint replacement. In spinal cord injured young people who are dependent upon their shoulders for their mobility, or who place additional stress on their hips because of an abnormal gait, avascular necrosis could be devastating.

Saisu recently reported a $27 \%$ incidence rate of osteonecrosis of the femoral head in renal transport patients receiving $0 \mathrm{mg}$ to $4000 \mathrm{mg}$ corticosteroid therapy and a $100 \%$ rate in those receiving more than $4000 \mathrm{mg}$ (total intravenous sustained and pulse dose) of Mpred (equivalents) in the first three months post transplant. ${ }^{7}$ Similarly, Marsh reported a $21 \%$ incidence of AVN in patients treated for aplastic anaemia with antilymphocyte globulin (ALG) followed by $5 \mathrm{mg} / \mathrm{kg} /$ day doses of Mpred (to treat the consequent serum sickness) in contrast to a $0 \%$ incidence using $1 \mathrm{mg} / \mathrm{kg} /$ day dosages. ${ }^{8}$ Patients in the Marsh study were treated for 5 days with ALG and between 2-4 weeks with Mpred. Continuous high-dose Mpred treatment was also found to be a risk factor for AVN in the treatment of systemic lupus erythematosus. ${ }^{9,10}$ Several case reports have shown humeral and femoral AVN resulting from short term, high dosage steroid treatments $(520-3400 \mathrm{mg}$ over 7 to 32 days $) .^{11-16}$ Felson and Anderson's metaanalysis of 22 previous AVN incidence studies comparing steroid dose and bolus steroids indicated that a $9000 \mathrm{mg}$ prednisone (equivalent) cumulative dosage given in a month had a $22 \%$ incidence of AVN. ${ }^{17}$

To investigate whether the NASCIS-2 Mpred protocol results in an increased incidence of AVN of the femoral or humeral head within 12 months of injury, we conducted a prospective cohort study of patients treated at two centres between 1989 and 1996.

\section{Method}

Potential subjects for the study were those patients with confirmed neurologic deficit (ASIA grade D or worse) admitted to the Acute Spinal Cord Injury Unit (ASCIU) of Vancouver's University Hospital (relocated to the Vancouver Hospital and Health Sciences Centre in 1994), and the Health Sciences Centre Rehabilitation Hospital (Winnipeg) between December 1988 and June 1995. All candidates had received treatment directed by a spinal cord injury physician within $8 \mathrm{~h}$ of injury. Within this group some received a $30 \mathrm{mg} \mathrm{kg}^{-1}$ loading dose of Mpred given over $15 \mathrm{~min}$, followed by a $45 \mathrm{~min}$ pause before beginning maintenance by a $5.4 \mathrm{mg} \mathrm{kg}^{-1} \mathrm{hr}^{-1}$ intravenous infusion for $23 \mathrm{~h},{ }^{5}$ while others treated before the publication of the NASCIS- 2 study or presenting more than $8 \mathrm{~h}$ post injury did not receive steroids.
Patients were excluded from the study if they had a factor likely to result in a false positive MRI scan: were younger than 15 years (to ensure closure of femoral capital epiphysis); or older than 75 years (advanced joint destruction due to aging); had any hip or shoulder disease with radiologic abnormality; or pelvic fracture. Patients were also excluded if they had a history predisposing to AVN, including hip dislocation, excessive alcohol consumption, previous high-dose steroid use (including sports related), or systemic lupus erythematosus.

Patients wider than $55 \mathrm{~cm}$ across the chest/ shoulders/hips or weighing more than $134 \mathrm{~kg}$ exceeded the capacity of our MRI machine and thus were ineligible for the study. Those suffering from claustrophobia or having free floating metal in their body (bullets, fragments in eye etc.) were also ineligible for MRI scanning. Because of budget constraints any potential subject requiring more than $\$ 500.00$ (Can) in transportation reimbursement was not enrolled.

Ethical approval (C91-069 for this study) was obtained from The University of British Columbia Clinical Screening Committee for Research Involving Human Subjects, as well as separately from the University of Manitoba. All subjects provided informed consent. A retrospective chart review identified eligible subjects who were then contacted by letter for participation in the prospective assessment for AVN.

A magnetic resonance imaging (MRI) scan of both humeral and both femoral heads was performed on each subject at a minimum of 6 months post-injury (mean 21.4, range 6-46). The two radiologists evaluating the MRI scans were unaware of the treatment protocol received by the individual subject. Coronal and axial T1 weighted (TR800 TE20) and T2 weighted (TR2000 TE20/80) images were obtained. Throughout the study the radiologists reported their findings by consensus.

Our population sample size requirements were based on extremely conservative AVN incident rate estimates of $2 \%$ in the control group and $10 \%$ in the treatment group, recognizing that there had been no prior report of the incidence of AVN in the spinal cord injured. By this criteria, 107 subjects were required in each group. Since most patients did not return to their pre-injury residence efforts to locate them included contacting next of kin (as recorded during hospital admission), family physicians, the Canadian Paraplegic Association, telephone services, rehabilitation services, and medical insurance services.

\section{Results}

Collecting the estimated 214 subjects was not possible due to poor patient compliance and lack of forwarding addresses. There were 508 potential subjects admitted to the ASCIU in Vancouver between December 1988 and June 1995. Of these, 253 met the inclusion criteria. We 
were unable to contact 82 of those eligible. Of the remainder only 62 agreed to enroll. The Winnipeg ASCIU enrolled 29 subjects all of whom were treated with high dose steroid protocols between July 1990 and August 1994. Budget constraints forced enrollment in the non-steroidal group to be closed contingent upon no case of AVN appearing to that time in the steroid group. (One patient was found to have AVN by MRI scan but, radiographic evidence revealed an older injury which the patient admitted having forgotten about; consequently, this patient was excluded from the study).

There were 32 spinal cord injured subjects (seven females, 25 males), aged 16-65 years (mean 34 years) who did not receive steroids. There were 59 spinal cord injured (five females, 54 males), aged 15-64 years (mean 32 years), who received the NASCIS-2 protocol.

Had a group with AVN been identified, statistical analysis would have been difficult; however, there was no case of AVN found in either group. Therefore, using binomial distribution, the true incidence of AVN among the methylprednisolone treated group is predicted to be less than $5 \%(\alpha<0.05)$.

\section{Discussion}

Because of the results of the NASCIS-2 study we could not ethically withhold Mpred treatment from one group of subjects. Therefore, retrospective enrollment of controls was necessary. Upon release from hospital, the cord-injured people seldom returned to their preinjury residence because of their new needs for building access, assisted living, and transportation convenience. Once located, many potential subjects chose not to participate because they wanted no reminders of the incident and recover process that had so drastically changed their lives. Of those who agreed to participate, many later either reneged or failed to attend for the MRI scan. Even invitation to participate while in the rehabilitation hospital failed (this would have still allowed MR scanning to be done at about three months after treatment). Consequently it was at least twelve months post injury before any of these candidates were assessed.

After the initial reporting of the NASCIS- 2 study, ${ }^{5}$ nursing and pharmacy reports ${ }^{6,18}$ described a formula for calculating the Mpred dosage which included an additional $2500 \mathrm{mg}$ Mpred for priming the intravenous administration set. Our study attached the Mpred solution bag to an existing intravenous delivery; therefore, we used the protocol as initially published. ${ }^{5}$ While there could be a difference in the total amount of Mpred delivered by the two methods $5,6,18$ we do not believe any consequent difference in neurologic improvement would alter the clinical question of whether the neurological benefit offset the risk of AVN. We felt that even a low incidence (e.g. $10 \%$ ) of AVN would be unacceptable considering its impact on the mobility of the spinal cord injured and the notoriously poor outcome of its treatment measures.
Why was AVN not seen in these patients, despite the very high steroid dose? We suggest that the steroid effect is only seen where vasculitis has been triggered by an immune reaction (to a serum or a grafted organ, or in autoimmune disease). This explains the baseline incidence of AVN in a number of these conditions, and the absence after spinal cord injury. This suggests that the use of megadose steroid will be safe if of short duration. The use of megadose mpred in a recently cord-injured person in whom such immune processes already exist may carry the risk of AVN, a risk that should be discussed with the patient.

Since no evidence of AVN was found, the incidence rate predicted by our sample size is less than $5 \%$ $(\alpha<0.05$, with a power of 0.8$)$, and we continue to recommend the use of the NASCIS-2 high dose methylprednisolone protocol, provided it is restricted to a $24 \mathrm{~h}$ period. The publication of the NASCIS-3 protocol suggesting that the methylprednisolone be continued for $48 \mathrm{~h}$ in certain patients is probably safe but a monitoring program may be required. ${ }^{19}$

\section{Acknowledgements}

We wish to thank the Rick Hansen Man in Motion Legacy Fund for its financial assistance in cooperation with The Alberta Paraplegic Foundation and The Canadian Paraplegic Association. (Grant \#91-45). We also wish to thank Leonard Tong, Senior MRI Technologist, Vancouver Hospital and Health Sciences Centre, for readily giving us his skill and guidance throughout the project.

\section{References}

1 Collins WF. A review and update of experimental and clinical studies of spinal cord injury. Paraplegia 1983; 21: 204-219.

2 Braugler JM, Hall ED. Current application of 'high-dose' steroid therapy for CNS injury. $J$ Neurosurg 1985; 62: 806-810.

3 Hall ED, Braughler JM. Non-surgical management of spinal cord injuries: a review of studies with the glucocorticoid steroid methylprednisolone. Acta Anaesth Belgica 1987; 38: 405-409.

4 Bracken MB et al. Methylprednisolone and neurological function 1 year after spinal cord injury. J Neurosurg 1985; 63: 704-713.

5 Bracken MB et al. A randomized, controlled trial of methylprednisolone or naloxone in the treatment of acute spinal cord injury. Results of the second National Acute Spinal Cord Injury Study. New Eng J Med 1990; 322: 1405-1411.

6 Leake GC, Pascale VJ, Alfano SL, Bracken MB. Methylprednisolone for acute spinal cord injury [letter]. Am J Hosp Pharm 1990 Sep; 47: $1977-1978$.

7 Saisu T et al. High incidence of osteonecrosis of femoral head in patients receiving more than $2 \mathrm{~g}$ of intravenous methylprednisolone after renal transplantation. Transplantation Proceedings 1996; 28: 1559 - 1560 .

8 Marsh JC et al. Avascular necrosis after treatment of aplastic anaemia with antilymphocyte globulin and high-dose methylprednisolone. Br J Haematol 1993; 84: 731 - 735 .

9 Massardo L et al. High-dose intravenous methylprednisolone therapy associated with osteonecrosis in patients with systemic lupus erythematosus. Lupus 1992; 1: $401-405$.

10 Migliaresi S et al. Avascular osteonecrosis in patients with SLE: relation to corticosteroid therapy and anticardiolipin antibodies. Lupus 1994; 3: 37-41. 
11 Osebold WR, Kody MH. Bilateral humeral head osteonecrosis following spinal cord injury: a case report illustrating the importance of adhering to the recommendations of the Second National Acute Spinal Cord Injury Study. Iowa Orthop J 1994; 14: $120-124$

12 O'Brien TO, Mack GO. Multifocal osteonecrosis after shortterm high-dose corticosteroid therapy. A case report. Cain Orthop 1992; 279: 176-179.

13 Anderson JM, Orth M, Helm MB. Multiple joint osteonecrosis following short-term steroid therapy: a case report. $J$ Bone Joint Surg 1982; 64A: 139

14 Avital F, Alon M, Weiss S, Zer-Aviv F. Avascular necrosis of bone following short-term dexamethasone therapy for brain edema: a case report. J Neurosurg 1984; 61: 983.
15 Sambrook PN, Hassall JE, York JR. Osteonecrosis after high dosage, short term corticosteroid therapy. J Rheumatol 1984; 11: 514.

16 Taylor LF. Multifocal avascular necrosis after short-term highdose steroid therapy: a report of three cases. J Bone Joint Surg 1984; 66B: 431.

17 Felson DT, Anderson JJ. Across-study evaluation of association between steroid dose and bolus steroids and avascular necrosis of bone. Lancet 1987; 18: 902.

18 Hilton G, Frei J. High-dose methylprednisolone in the treatment of spinal cord injuries. Heart Lung 1991; 20: 675-680.

19 Bracken MB et al. Administration of methylprednisolone for 24 or 48 hours or tirilazad mesylate for 48 hours in the treatment of acute spinal cord injury. JAMA 1997; 277: $1597-1604$. 